\title{
Arrow-Shot Injury to Pediatric Spinal Cord
}

\author{
Vijayveer Singh ${ }^{1}$ Sharad Thanvi ${ }^{1}$ \\ ${ }^{1}$ Department of Neurosurgery, Dr. Sampurnanand Medical College, \\ Jodhpur, Rajasthan, India
}

Indian J Neurotrauma 2022;19:105-107.

\begin{abstract}
Address for correspondence Vijayveer Singh, Mch, Department of Neurosurgery, Dr. Sampurnanand Medical College, Room No. 111, Family Hostel, MDM Hospital Campus, Jodhpur 342003, Rajasthan, India (e-mail: Vvs8956@gmail.com).
\end{abstract}
Abstract
Keywords
- Brown-Sequard syndrome
- cervical spinal cord
- hemilaminectomy
- methylprednisolone
- pediatric population

Penetrating spinal cord injuries (PSCl) in cervical region are extremely rare in pediatric population. Most injuries in pediatric population are accidental due to gunshot or a stab injury with a sharp or pointed object. Gun shots may result into a severe wound which is usually fatal and may result in death, quadriplegia, or serious long-term disability. Stab injuries are less severe and may result in neurological sequalae. In this paper, an unusual case of pediatric arrow shot partial cervical cord injury is reported which was managed by aggressive neurosurgical management. The arrow lodged in the cervical cord was very near to the vertebral artery leading to parapariesis which recovered well without any complications. Diagnostic imaging at admission included radiographs, computed tomography (CT), and CT angiography of the cervical region. The patient underwent early surgical intervention with removal of foreign body from the cord and subsequent dural suturing.

\section{Introduction}

Pediatric arrow-shot injury with penetrating spinal cord injuries (PSCI) is rare ${ }^{1}$ and among the PSCI reported in literature, low thoracic and lumbar dominate the location. Cauda equina is the second most common location. Injuries to cervical cord are very rare and reported as unusual location which in most cases have fatal prognosis. ${ }^{2}$ Common penetrating agents are pencils, wooden splinters, bicycle spokes, and knives ${ }^{3}$.

Clinical features of cervical PSCI result directly from trauma but may also be associated with vascular impairment, which has been reported in autopsies. ${ }^{4}$ Depending on the exact site of injury, the most common neurological deficit includes high or low tetraplegia or end up with deep unilateral paresis. ${ }^{5}$ When a cutting edge runs between the spinous and transverse process, it may result in Brown-Sequard syndrome. ${ }^{6}$

\section{Case Report}

A 5-year-old female child presented to emergency room (ER) after she had been shot with an arrow by unknown person while at her home compound. The arrow stuck in the posterior surface of her neck (nuchal region) about the level of $\mathrm{C7}$ vertebra on the left side; which was not removed by parents and they immediately rushed her to nearby primary health center. After giving primary treatment and antitetanus prophylaxis, the child was referred to tertiary care center at Mathura Das Mathur Hospital, where she was admitted in ENT (ear-nose-tongue) department. After initial stabilization, she was referred to neurosurgery for further management. X-ray and computed tomography (CT) scan (with three-dimensional [3D] reconstruction image) of cervical region was done. While positioning the patient special precautions were taken to mobilize the spine as minimal as possible. Her head and neck were supported with hands while positioning her in supine position with neck tilted to right side for X-ray and CT scan. Her vitals were monitored throughout her time in the CT scanner. At the completion of the CT scanning, she was very gently turned to right semiprone position to avoid dislodgement of the arrow. X-ray showed an arrow stuck at C6-C7 vertebra level traversing left to right crossing
DOI https://doi.org/ $10.1055 / \mathrm{s}-0041-1724142$ ISSN 0973-0508.
(C)2021. Neurotrauma Society of India.

This is an open access article published by Thieme under the terms of the Creative Commons Attribution-NonDerivative-NonCommercial-License, permitting copying and reproduction so long as the original work is given appropriate credit. Contents may not be used for commercial purposes, or adapted, remixed, transformed or built upon. (https://creativecommons.org/licenses/by-nc-nd/4.0/).

Thieme Medical and Scientific Publishers Pvt. Ltd. A-12, 2nd Floor Sector 2, Noida-201301 UP, India 
the midline. CT scan of cervical region demonstrated that the arrow was transecting $\mathrm{C} 7$ lamina and traversing the spinal canal reaching up to right $\mathrm{T} 1$ pedicle from left-to-right sitting just short of the vertebral artery but without any damage to the vessel.

On clinical examination, the child had findings consistent with Brown-Sequard syndrome on right side. She was given methyl prednisolone infusion according to National Acute Spinal Cord Injury Studies (NASCIS) protocol ${ }^{7}$ and then transported to the operating room. In the operating room with due care and immobilization protocol, the child was shifted to operating table, kept in right lateral position and intubation was done. After intubation, she was placed in prone position exposing the arrow and nuchal region. Head was then fixed using elastic adhesive tapes and surgery was proceeded. Vertical midline skin incision was made and paraspinal and upper back muscles were dissected reaching up to lamina of $\mathrm{C} 7$ (the entry point of arrow in spinal canal). C7 hemilaminectomy was done, arrow was found piercing dura and entering the spinal cord. Dura was cut open on either side for $0.5 \mathrm{~cm}$ and a closed inspection for any cord injury was done, and it was found that arrow was piercing the cord tissue. Arrow was removed by gentle traction from the spinal canal, there was contusion of cord tissue but no blood oozing. The arrow was approximately $15 \mathrm{~cm}$ in length and $1 \mathrm{~cm}$ in diameter. There was oozing from bony elements which was controlled with bone wax. Dura was repaired by muscle patch, as there was loss of dural element to assure no cerebrospinal fluid (CSF) leak. Proper hemostasis was achieved and after assuring no CSF leak, closure was done layer wise.

The child was then shifted to surgical ICU for monitoring and was treated with intravenous (IV) fluids for maintenance and injection meropenem and methylprednisolone. She remained hemodynamically stable throughout the night and was shifted to ward after 18 hours. Postoperatively, the child had grade $4 / 5$ right lower extremity weakness. Postoperative recovery continued to be uneventful without any fever or CSF leak. Postoperative magnetic resonance imaging (MRI) could not be done because of poor financial status of the family (MRI in our center is under private sector which is charged). She was monitored intensively throughout her stay and discharged on 10th postoperative day with a plan for intensive neurosurgical follow-up and advised for physiotherapy and occupational therapy (-Fig. 1).

\section{Discussion and Literature Review}

Pediatric arrow-shot injuries are mostly accidental and in India occur mostly in tribal belts where children are often hit as a bystander. The clinical spectrum depends upon the anatomical location of the arrow shot. The current patient at the time of presentation was transported to our center within 6 hours of the shot. At the time of arrival in the ER, she was having stable cardiopulmonary parameters. The biggest challenge, however, was to image her with the arrow sitting in her neck and a high possibility of it being dislodged into a more critical position worsening her clinical status. A review of the literature from the experience as reported in cases with an intact arrow in the cervical vertebral column and also gunshot injuries was done. It was clear from this review that owing to the location of the arrow and the vicinity of the arrowhead with major vessels and the cord, both imaging and surgery was a serious challenge. This was achieved by the radiology, anesthesiology, and neurosurgery team to provide optimum position in the scanner. Cervical $\mathrm{X}$-ray in this patient became the initial reference showing foreign body stuck within the vertebral canal. CT scan facilitated assessment of range of injury and residual broken bony fragments allowing us to track a possible trajectory of penetrating agent. ${ }^{6,8}$

Many centers take up early exploration of these conditions based on results of the X-ray and CT before worsening of neurological condition. In the current patient, we were concerned regarding any deterioration in her clinical condition due to delay in surgery. But a review of the literature and also based on our experience in surgical exploration of traumatic cervical spine injuries, we decided to perform CT angiography with 3D reconstruction to evaluate exact neurosurgical pathology. This step proved to be crucial in the management of this patient because we knew that the arrow head was in a very crucial vicinity to vertebral vessels. We planned surgical plane and approach based on this imaging and had a better outcome in our patient without any intraoperative complications.

\section{Conclusion}

We therefore conclude that in such cases, a multidisciplinary team including neurosurgeon, radiologist, anesthesiologist, and pediatric neurologist must be in the driving seat right

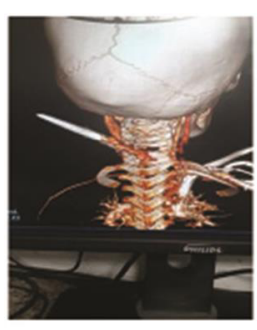

3D CT image PA view

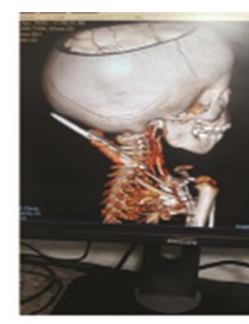

Lateral view

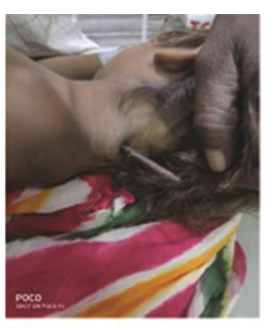

Pre-op image
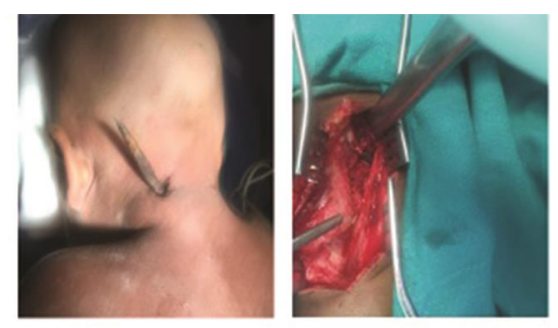

Position in OT
Intra- op image

Fig. 1 Pre-op and intra-op images along with radiological images. 3D, three-dimensional; CT, computed tomography; Intra-op, intraoperative; OT, operation theatre; PA, posteroanterior; Pre-op, preoperative. 
after the triage of the patient. Imaging must be planned to keep in view that manipulation of the head and neck and sometimes the trunk will be limited because of the arrow and patient may require anesthetic expertise for sedation and positioning.

CT angiography should be included in the evaluation of these patients to reduce intraoperative complications and, also to improve overall outcome. All patients must be given antitetanus prophylaxis depending upon their vaccination status and treated with broad spectrum antibiotics to reduce the chances of CNS infection, especially meningitis.

\section{Conflict of Interest}

None declared.

\section{References}

1 Al-Janabi T, Nayeem N, Smallman W. Stab wound to the neck-a rare presentation. Eur J Emerg Med 2001;8(1):55-56

2 Benzel EC, Hadden TA, Coleman JE. Civilian gunshot wounds to the spinal cord and cauda equina. Neurosurgery $1987 ; 20(2): 281-285$
3 Roach MJ, Chen Y, Kelly ML. Comparing blunt and penetrating spinal cord injury. Analysis of long term functional and neurological outcomes. Top Spinal Cord Inj Rehabil 2018;24(2):121-132

4 Kramer MM, Acker A, Ohana N, Penetrating spinal cord injury. In: Dionyssiotis Y. Essentials of Spinal Cord Injury Medicine. London, United Kingdom: Intechopen; 2018: 564

5 Copley PC, Tilliridou V, Kirby A, Jones J, Kandasamy J. Management of cervical spine trauma in children. Eur J Trauma Emerg Surg 2019;45(5):777-789

6 Rajmohan B. Brown-Séquard syndrome following stab injury. ANZ J Surg 2006;76(8):760-762

7 Snacker MB, Sheperd MJ, Collin Wf, et al. A random control trial of methiprednisolone or naloxone in treatment of acute spinal cord injury. Res Second NASCIS N Eng J Med 1990;322:1405-1411

8 Bonnes S, Smith C. Initial managementof acute spinal cord injury. Contin Educ Anaesth Crit Care Pain 2013;13(6):224-231 